\title{
In vivo fluorescence spectroscopic monitoring of radiotherapy in cancer treatment
}

\author{
Seung Hee Han, ${ }^{1}$, Tai-Kyong Song ${ }^{3}$ \\ ${ }^{1}$ Sogang Institute Advanced Technology, Sogang University, Seoul, Korea \\ ${ }^{2}$ Division of Biophysics and Bioimaging, Ontario Cancer Institute, University Health Network, Toronto Ontario, Canada \\ ${ }^{3}$ Department of Electronic Engineering and Interdisciplinary Program of Integrated Biotechnology, Sogang University, Seoul, Korea
}

Received September 17, 2014; Revised November 21, 2014; Accepted November 24, 2014; Published Online November 29,2014

\section{Original Article}

\begin{abstract}
Purpose: The primary purpose of this study is to introduce fluorescence spectroscopy technology as a tool for optical biopsy and to evaluate the reliability of this real-time non-invasive technique in assessing tumor therapeutic response in radiotherapy. Methods: Four oral cancer patients with inflammatory conditions of the oral cavity volunteered to participate in the study. Using FastEEM (Excited Emission Matrix), spectra were taken from both normal and tumor regions. Then the second optical diagnostic was taken from the tumor regions in 4 patients with time delay of 4 weeks. The fluorescence spectroscopy optical biopsy turned out to be more suitable for tumor diagnostics as there was a significant difference in fluorescence spectra. Results: The first fluorescence intensity of cancerous tissue was higher compared to the second fluorescence intensity of optical biopsy of cancerous oral tissue. From this result, it can be concluded that the fluorescence spectroscopy optical biopsy, which is a technique that does not require removal of tissue sample from body, is a reliable tool to be used in radiotherapy. Conclusion: FastEEM, which is a tool for fluorescence spectroscopy, enables a real-time non-invasive assessment of the chemical, biological, as well as morphological variations in the tissue composition following radiotherapy.
\end{abstract}

Keywords: Radiotherapy; Fluorescence Spectroscopy; Tissue Diagnosis; Oral Cancer; Monitoring

\section{Introduction}

The outcomes of cancer treatments such as radiotherapy, photodynamics therapy, chemotherapy, and gene-therapy depend on factors like tumor type, tumor stage, and patient health. For advanced-stage tumors, neoadjuvant therapies such as chemotherapy, radiation therapy, and combination therapy (i.e. chemo-radiation therapy) are often used to decrease tumor size before surgery. Since those therapies are often used before conducting surgeries, tumor resection and organ preservation are feasible. Sometimes patients gain complete recovery from neoadjuvant therapies. On the other hand, some patients do not exhibit favorable responses to such treatment. These varied responses result from differences in tumor functional parameters across the patient pool. The effectiveness of cancer treatment is judged mainly by the extent of reduction in tumor size. However, tumor functional changes (e.g. changes in tumor metabolism) from treatment often precede gross structural changes. Thus methods sensitive to tumor functional changes are of interest for assessing treatment efficacy early and for adjusting the treatment plan accordingly., ${ }^{1,2}$

Optical spectroscopic diagnostics allow non-invasive physical and chemical characterization of biological tissues..$^{3,4}$ The structural and chemical composition of cells and tissues strongly influence their optical features, and therefore, alterations in the optical characteristics may indicate the presence of diseased tissue. Optical spectroscopy may provide possibilities in the early detection of cancerous tissues in humans. Biochemical and structural or morphological information can be gained by measuring absorption, (auto) fluorescence, elastic scattering, or Raman scattering. These spectroscopic techniques have distinct physical bases and each have the potential to become a more advanced conventional cancer detection method when combined to form an adjuvant method. ${ }^{3-5}$ Nonetheless, of all these optical techniques, spectroscopy of tissue auto-fluorescence has been considered to be the most promising.

Laser-induced fluorescence (LIF) is a real-time spectroscopic technique that can be used to characterize biomedical and morphological changes of different kinds of tissue both in vitro $^{6}$ and non-invasively in vivo. In vitro fluorescence measurements are usually recorded from an area of the tissue surface that is larger than the size of the excitation beam. Most clinical applications of fluorescence spectroscopy require that spectra be recorded remotely via optical fibers. 
The fluorescence is therefore collected from a relatively smaller area of the tissue surface. ${ }^{7,8}$ The sampling depth for fluorescence measurement is determined by the penetration depth of the excitation light, the various fluorescence coefficients of the tissue layers, the escape functions for the fluorescence, as well as the detection geometry. ${ }^{8}$

It is often the shape of the fluorescence spectra, and not the fluorescence intensity, that is used for diagnostic purposes. The difference in excitation and/or emission spectra between normal and malignant tissues can thus be an important tool for tissue diagnostics. This makes the measurement slightly less dependent on the detection geometry, the exact excitation power, and the detector sensitivity.

Fluorescence that is obtained from endogenous tissue fluorophores without any external fluorophore added is called autofluorescence. ${ }^{9}$ Alterations in tissue composition from normal to malignant tissue form a basis for the diagnostic potential for demarcating neoplastic tissue. ${ }^{10}$ Tissue fluorophores will normally fluoresce in the visible wavelength range following excitation with radiation in the UV and near-UV region. The auotofluorescence intensity is frequently lower in tumor tissues compared to that in the surrounding normal tissues. ${ }^{11,12}$ The endogenous tissue fluoropores can be divided into two main groups; one group involved in the cellular energy metabolism and the other in the structural proteins. ${ }^{3}$ The most important tissue fluorophores are collagen, elastin, and NADH (Nicotinamide adenine dinucleotide)/NADPH (Nicotinamide adenine dinucleotide phosphate)..$^{13,14}$ All fluorescence spectra recorded from the tissues are also influenced by the optical properties of the tissues. This means that those absorbers do not fluoresce, but still absorb either the excitation or fluorescent light, ultimately affecting the overall intensity of the fluorescence spectra. The shape of the spectra is usually less affected as the absorption coefficient in the visible region is lower than that of the excitation light in the violet or near-UV region. ${ }^{14}$

Figure 1 shows the fluorescence emission spectra of tissue fluorophores after being excited with $337 \mathrm{~nm}$ light. It compares the fluorescence spectra obtained from a pre-malignant lesion and the normal surrounding tissue in vivo after administration of ALA (Aminolevuliniv acid). The wavelength of the excitation light was $405 \mathrm{~nm}$ and the dual emission peaks from PpIX (Protoporphyrin IX) were obtained at 635 $\mathrm{nm}$ and $705 \mathrm{~nm}$.

Ionizing radiation in radiotherapy causes atoms and molecules in tissues to become ionized or excited. This can ultimately produce free radicals, break chemical bonds, form new chemical bonds and cross-linkage between macromolecules, or damage molecules that regulate vital cell processes (e.g. DNA, RNA, proteins). In cases of intensive radiation, cells would not be replaced fast enough and tissues will fail to function.

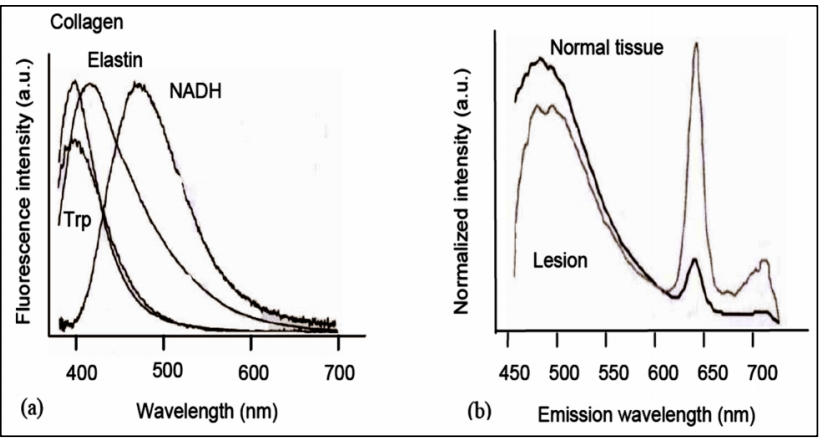

FIG. 1: Fluorescence spectra; (a) from some important tissue fluorophores following $337 \mathrm{~nm}$ excitation, (b) typical average fluorescence spectra obtained in vivo from normal and premalignant lesion on vocal folds, using $405 \mathrm{~nm}$ excitation light where ALA has been applied prior to the examination. Modified from Ref (Eker C). ${ }^{4}$

To better understand the clinical responses of tumors to radiation therapy, experiments need to shift from individual cell to tissue level. This understanding will in turn allow the application of clinical knowledge to cancer treatment to monitor therapies. In clinical radiation therapy, the dose of radiation that can be applied is limited, because of its damage on the surrounding normal tissues. The efficacy of the therapy can be improved both through more efficient radiation delivery to the tumor with minimization of normal tissue damage and through increased sensitivity of the tumor to radiation. First, effective radiation delivery to the tumor is achieved by better $\mathrm{X}$ - and $\gamma$-ray treatment machines. They use a better arrangement of source distribution so that even deep human tumors (compared to those at the surface) can be treated effectively without too much normal tissue damage. To increase the sensitivity of the tumor, however, one first needs to understand the difference in biological responses of tumors and normal tissues. The deployment of fractionated radiation, which involves 1-2 Gy dosage a day over a total of 5 to 7 weeks, is one of the most important developments in this respect. During fractionation, dosage can be adjusted based on the tumor response as it is done during accelerated radiation therapy. Furthermore, increasing oxygen sensitivity and modulating the vascular response can lead to improved treatment efficacy.

\section{Methods and Materials}

\section{Patients}

Four volunteer oral cancer patients with a known or suspected premalignant or malignant oral cavity lesion were recruited from the Department of Oral Cancer Center at the Medical School, University of Hallym. Informed consent was obtained from each person.

\section{Instrument}

An instrument for acquisition and analysis of tissue fluorescence in a clinical setting must meet several requirements. Since native tissue fluorescence (auto fluorescence) is weak, 
sensitive detection and strong excitation are required. Data acquisition must provide a good $\mathrm{S} / \mathrm{N}$ ratio, but it should also be rapid enough to avoid motion artifacts and not interfere with the routine clinical procedure. In addition, a wide range of fluorescence excitation wavelength must be available in order to provide complete information about the optical properties of the tissue sample. Furthermore, the components must be able to be interfaced with rapid on-line data analysis software. Finally, the system must require little maintenance, be portable, be easy to use, and be compact. These design issues were key factors taken into account when designing FastEEM.

The spectroscopic system used to measure fluorescence EEMs shown in Figure 2 measures fluorescence emission spectra at 11 excitation wavelengths, within the range of 308 to $505 \mathrm{~nm}$. The system incorporates a fiber-optic probe and two kinds of light sources; dye laser pumped by a nitrogen laser (337 nm, model VSL-337MD; Laser Science, Inc) laser and a xenon arc lamp (EG\&G Optoelectronics, FX-139). The dye laser consists of ten cuvettes that are mounted on a wheel driven by a motor rotating at $5 \mathrm{~Hz}$. The fluorescence spectra of the dye laser used ranged from $350 \mathrm{~nm}$ to $650 \mathrm{~nm}$ and the emission power correlated with the wavelength of the dye. The power was always less than $20 \mu \mathrm{J}$. The xenon arc lamp is for white light reflectance spectrum from 300 to $800 \mathrm{~nm}$ and is coupled with a monochromator to provide excitation light. It is also connected to a cooled Intensity charge-coupled device (ICCD) camera (Max 1024; EG\&G Instruments Princeton Applied Research) that records fluorescence intensity as a function of emission wavelength. Fluorescence emission as well as reflectance signals were collected by the fiber-optic probe consisting of seven multimode optical fibers with diameters of $1.5 \mathrm{~mm}$. They were coupled to a scanning imaging spectrograph (CP 200, Jobin Yvon SA, Edison, NJ) fitted with a high-sensitivity ICCD array. The EEM collection time was optimized in less than 1 second through the ICCD-fitted spectrograph with multiple emission intensities being read simultaneously. The excitation wavelength range was extended to $308-505 \mathrm{~nm}$ and emission was collected between the wavelengths of 300 and $700 \mathrm{~nm}$.

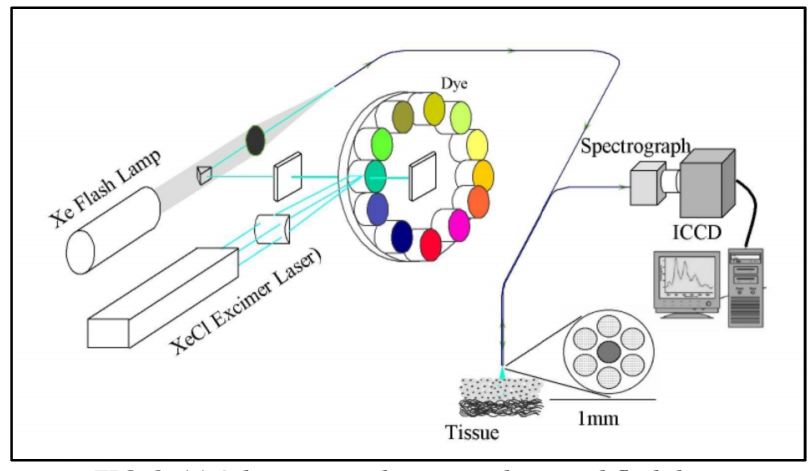

FIG. 2: (a) Schematic with excimer laser and flash lamp

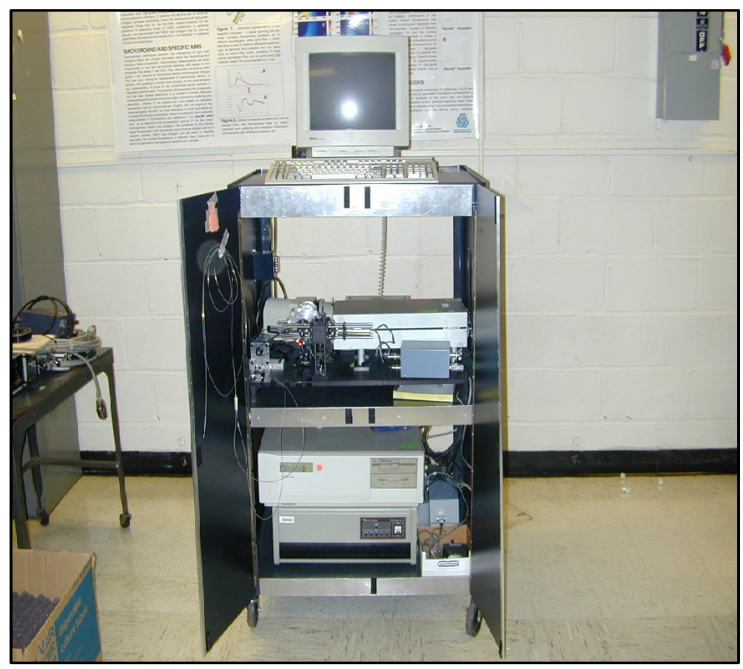

FIG. 2: (b) Photograph of FastEEM.

\section{Calibration}

Thorough calibration of the system was performed prior to data collection. A mercury lamp was used to calibrate the wavelength scale of the ICCD detector. A NIST standard tungsten lamp provided spectral response calibration (primarily determined by the spectrograph grating and ICCD detector). A spectralon disk (Labsphere) with approximately $20 \%$ reflectance was used for white light reflectance calibration to correct for the xenon flash lamp spectral line shape and intensity. Fluorescence intensity correction was achieved using a cuvette with a standardized solution of rhodamine B in thylene glycol. This allowed for a comparison of tissue fluorescence intensities without day-to-day intensity fluctuations that result from small variations in system alignment. Furthermore, by measuring the Rhoda mine fluorescence response on a well calibrated spectrofluorimeter at different excitation wavelengths, FastEEM system was calibrated to account for variations in laser power at different excitation wavelengths.

Finally, a background spectrum for all excitation wavelengths was recorded and was subtracted from the patient data to eliminate the offset resulting from dark counts of the ICCD detector and fluorescence and reflectance background created by the optical fiber probe.

The spectralon, Rhodamine, and background calibration data were stored in the system and were used for automatic calibration and display of the data. Additionally, these files were used in data processing to obtain an on-line diagnosis. Several concentrations of different fluorophores were measured to determine the sensitivity and response of the system over a large wavelength range.

\section{Measurement}

CT (Computed Tomography) and MRI (Magnetic resonance imaging) scans provided additional information about the location and size of each tumor. FastEEM measurements were 
carried out consecutively. The pre-radiation measurements were used as baseline data. Subsequently, weekly measurements were carried out for each individual until the treatment was completed. Each patient received daily fractionated irradiation from Monday through Friday, and the optical fluorescence spectroscopic measurements were completed just before treatment began each week. Daily fractionated radiotherapy was administrated with a $3 \mathrm{D}$ conformal radiotherapy technique. A simultaneous in-field boost prescription technique was used for prescribing $50 \mathrm{~Gy}$ in $2.5 \mathrm{~Gy}$ per fraction over 20 fractions for both primary and gross neck disease. The duration of treatment was about 4 weeks. The standard RECIST (Response Evaluation Criteria In Solid Tumors) was applied to classify tumor responses. ${ }^{15} \mathrm{~A}$ responder is defined as a patient with no evidence of residual cancer in the neck dissection specimen at the end of treatment. Formal assessment of treatment response was conducted 4 weeks after completing the therapy. Post-radiotherapy neck dissections were evaluated using a pathologic response.

\section{Data analysis}

In this part of the study, software was developed to perform data analysis in real time. Fluorescence and reflectance spectra have been analyzed using extensive modeling of light-tissue interactions to provide three types of spectroscopic information; Intrinsic Fluorescence Spectroscopy (IFS), Diffuse Feflectance Spectroscopy (DRS), and Light Scattering Spectroscopy (LSS). ${ }^{16-18}$ In the IFS analysis, information from measured fluorescence and reflectance spectra were combined using a photon migration-based model. The model was used to extract the intrinsic tissue fluorescence that is free from distortions introduced by tissue scattering and absorption. Intrinsic tissue fluorescence spectra were then decomposed into a linear combination of fluorescence contributions from collagen and $\mathrm{NADH}$, or other diagnostically important chromosphores. ${ }^{5}$ Thus we extracted quantitative biochemical information that can be compared to a specific pre-determined threshold to classify the examined tissue site as normal or diseased. The measured reflectance spectra were processed using DRS and LSS analysis to obtain information from multiply and singly backscattered light.

In the LSS step, the spectral features of the multiply scattered light determined by the diffusion model analysis were subtracted from the measured reflectance. This provided the spectrum of light that is backscattered from the tissue after undergoing a single scattering event. Analysis of this spectrum yielded information about the size distribution and number density of the nuclei of epithelial cells. The values of these parameters were compared to pre-established threshold values to determine whether the light scattering characteristics of the examined tissue sites are consistent with normal or diseased tissue. In the final step of the analysis, information from the IFS, DRS, and LSS analyses were combined to form a consensus diagnosis. ${ }^{5}$ This appears in the LABVIEW operator control window. The entire data analy- sis process took 10 seconds using an Intel Celeron processor, but can be expected to be significantly faster (3 seconds) when a faster computer is used.

\section{Results}

In this experiment, we tried to obtain two types of data to monitor tumor radiotherapy. The first was the response of the blood and the other was the spectrum analysis in the fluorescence spectroscopy.

TABLE 1: Characteristics of patients with oral cancer (SCC = squamous cell carcinoma, TNM = Tumor, Node, Metastasis stage

\begin{tabular}{cccccc}
\hline $\begin{array}{c}\text { Patient } \\
\text { No }\end{array}$ & Age/Sex & $\begin{array}{c}\text { Histologic } \\
\text { Type }\end{array}$ & $\begin{array}{c}\text { TNM } \\
\text { stage }\end{array}$ & $\begin{array}{c}\text { Size/Depth } \\
(\mathrm{cm})\end{array}$ & $\begin{array}{c}\text { Treatment } \\
\text { response }\end{array}$ \\
\hline P-1 & $58 / \mathrm{F}$ & SCC & T4N2bM0 & $4.1 \times 2 / 0.4$ & complete \\
P-2 & $61 / \mathrm{M}$ & SCC & T2N2bM0 & $2.1 \times 2.4 / 0.3$ & complete \\
P-3 & $50 / \mathrm{M}$ & SCC & T4N2cM0 & $3.6 \times 3.1 / 0.5$ & complete \\
P-4 & $63 / \mathrm{M}$ & SCC & T2N2cM0 & $3.3 \times 2.8 / 0.3$ & complete \\
\hline \hline
\end{tabular}

TABLE 2: Individual tumor $(\mathrm{t})$ and normal tissue $(\mathrm{n})$ relative blood flow changes $(\mathrm{rBF}(\%))$ at the end of week-1, week-2, week-3 and week -4 of radiotherapy. Pretreatment value at week- 0 was defined as $100 \%$ in all patients.

\begin{tabular}{cccccc}
\hline \hline $\begin{array}{c}\text { Patient } \\
\text { No }\end{array}$ & $\begin{array}{c}\text { Tissue } \\
\text { type }\end{array}$ & $\begin{array}{c}\text { Week-1 } \\
(\%)\end{array}$ & $\begin{array}{c}\text { Week-2 } \\
\text { (\%) }\end{array}$ & $\begin{array}{c}\text { Week-3 } \\
\text { (\%) }\end{array}$ & $\begin{array}{c}\text { Week-4 } \\
\text { (\%) }\end{array}$ \\
\hline P-1 & $\mathrm{t}$ & $150 \pm 4$ & $126 \pm 3$ & $92 \pm 2$ & $113 \pm 6$ \\
P-1 & $\mathrm{n}$ & $101 \pm 6$ & $112 \pm 4$ & $103 \pm 4$ & $113 \pm 5$ \\
P-2 & $\mathrm{t}$ & $162 \pm 7$ & $152 \pm 4$ & $167 \pm 2$ & $123 \pm 7$ \\
$\mathrm{P}-2$ & $\mathrm{n}$ & $93 \pm 4$ & $98 \pm 6$ & $89 \pm 3$ & $101 \pm 1$ \\
$\mathrm{P}-3$ & $\mathrm{t}$ & $114 \pm 7$ & $132 \pm 4$ & $144 \pm 1$ & $119 \pm 12$ \\
$\mathrm{P}-3$ & $\mathrm{n}$ & $85 \pm 2$ & $89 \pm 8$ & $95 \pm 4$ & $92 \pm 7$ \\
$\mathrm{P}-4$ & $\mathrm{t}$ & $156 \pm 4$ & $136 \pm 1$ & $114 \pm 6$ & $166 \pm 3$ \\
$\mathrm{P}-4$ & $\mathrm{n}$ & $107 \pm 1$ & $99 \pm 4$ & $87 \pm 2$ & $82 \pm 9$ \\
\hline \hline
\end{tabular}

TABLE 3: Weekly blood oxygen saturation $\left(\mathrm{StO}^{2}\right)$ (\%) changes during radiotherapy for both tumor $(\mathrm{t})$ and normal tissue $(\mathrm{n})$.

\begin{tabular}{ccccccc}
\hline \hline $\begin{array}{c}\text { Patient } \\
\text { No }\end{array}$ & $\begin{array}{c}\text { Tissue } \\
\text { type }\end{array}$ & $\begin{array}{c}\text { Week-0 } \\
(\%)\end{array}$ & $\begin{array}{c}\text { Week-2 } \\
(\%)\end{array}$ & $\begin{array}{c}\text { Week-3 } \\
(\%)\end{array}$ & $\begin{array}{c}\text { Week-4 } \\
(\%)\end{array}$ & $\begin{array}{c}\text { Week-4 } \\
(\%)\end{array}$ \\
\hline P-1 & $\mathrm{t}$ & $54 \pm 4$ & $51 \pm 3$ & $52 \pm 2$ & $48 \pm 6$ & $48 \pm 4$ \\
P-1 & $\mathrm{n}$ & $56 \pm 5$ & $52 \pm 4$ & $57 \pm 4$ & $53 \pm 2$ & $52 \pm 6$ \\
P-2 & $\mathrm{t}$ & $62 \pm 7$ & $58 \pm 4$ & $57 \pm 3$ & $63 \pm 7$ & $61 \pm 7$ \\
$\mathrm{P}-2$ & $\mathrm{n}$ & $53 \pm 4$ & $61 \pm 2$ & $60 \pm 3$ & $67 \pm 1$ & $64 \pm 4$ \\
$\mathrm{P}-3$ & $\mathrm{t}$ & $64 \pm 1$ & $62 \pm 7$ & $66 \pm 1$ & $59 \pm 12$ & $61 \pm 4$ \\
$\mathrm{P}-3$ & $\mathrm{n}$ & $68 \pm 2$ & $63 \pm 8$ & $71 \pm 4$ & $67 \pm 7$ & $66 \pm 2$ \\
$\mathrm{P}-4$ & $\mathrm{t}$ & $63 \pm 4$ & $66 \pm 1$ & $64 \pm 6$ & $73 \pm 3$ & $69 \pm 4$ \\
$\mathrm{P}-4$ & $\mathrm{n}$ & $67 \pm 1$ & $75 \pm 4$ & $69 \pm 2$ & $77 \pm 9$ & $73 \pm 1$ \\
\hline \hline
\end{tabular}

A total of 4 oral cancer patients were examined weekly. The patient and tumor characteristics are given in Table 1. Tables 2 to 4 summarize the data from tumor $(\mathrm{t})$ and normal tissue (n) of 4 patients. Table 2 exhibits $r B F$ (relative Blood Flow) [\%] at the end of week-1, week-2, week-3 and week-4 of radiation therapy. The pre-treatment value at week- 0 was 
defined as $100 \%$ in all patients. Similarly, Table 3 and Table 4 summarize weekly changes of $\mathrm{StO}^{2}$ (oxygen saturation) [\%] and THC (total hemoglobin concentration) $\left[{ }^{1} \mathrm{M}\right]$, respectively. This has also been the case in animal experiments and clinical trials that employed radiation therapy; apparently this variation is only partly a result of methodological factors such as differences in probe handling and positioning on the tissue. ${ }^{1,19}$

TABLE 4: Weekly total hemoglobin concentration (THC) ( $\left.{ }^{1} \mathrm{M}\right)$ changes during chemoradiation therapy for both tumor $(\mathrm{t})$ and normal tissue (n).

\begin{tabular}{ccccccc}
\hline \hline $\begin{array}{c}\text { Patient } \\
\text { No }\end{array}$ & $\begin{array}{c}\text { Tissue } \\
\text { type }\end{array}$ & $\begin{array}{c}\text { Week-0 } \\
(\mathrm{mM})\end{array}$ & $\begin{array}{c}\text { Week-2 } \\
(\mathrm{mM})\end{array}$ & $\begin{array}{c}\text { Week-3 } \\
(\mathrm{mM})\end{array}$ & $\begin{array}{c}\text { Week-4 } \\
(\mathrm{mM})\end{array}$ & $\begin{array}{c}\text { Week-4 } \\
(\mathrm{mM})\end{array}$ \\
\hline $\mathrm{P}-1$ & $\mathrm{t}$ & $84 \pm 4$ & $71 \pm 3$ & $72 \pm 2$ & $68 \pm 6$ & $58 \pm 4$ \\
$\mathrm{P}-1$ & $\mathrm{n}$ & $76 \pm 2$ & $69 \pm 7$ & $75 \pm 3$ & $69 \pm 2$ & $63 \pm 6$ \\
$\mathrm{P}-2$ & $\mathrm{t}$ & $72 \pm 7$ & $68 \pm 3$ & $77 \pm 1$ & $73 \pm 6$ & $71 \pm 9$ \\
$\mathrm{P}-2$ & $\mathrm{n}$ & $73 \pm 8$ & $75 \pm 2$ & $80 \pm 1$ & $77 \pm 9$ & $84 \pm 2$ \\
$\mathrm{P}-3$ & $\mathrm{t}$ & $64 \pm 1$ & $62 \pm 7$ & $66 \pm 1$ & $59 \pm 12$ & $61 \pm 4$ \\
$\mathrm{P}-3$ & $\mathrm{n}$ & $78 \pm 2$ & $83 \pm 2$ & $81 \pm 2$ & $87 \pm 1$ & $86 \pm 2$ \\
$\mathrm{P}-4$ & $\mathrm{t}$ & $53 \pm 4$ & $46 \pm 9$ & $51 \pm 6$ & $49 \pm 3$ & $55 \pm 4$ \\
$\mathrm{P}-4$ & $\mathrm{n}$ & $61 \pm 1$ & $55 \pm 2$ & $62 \pm 7$ & $57 \pm 9$ & $66 \pm 5$ \\
\hline \hline
\end{tabular}

The biological significance of an increase in tumor blood flow is not well understood. It is possible that the increase may improve tumor oxygenation, which in turn might improve tumor radiosensitivity. ${ }^{20}$ The mechanism for such a favorable response might be reflected in the observations of Sonveaux. ${ }^{2}$ These investigators concluded that clinically relevant doses of radiation elicit a vascular stress response with increased secretion of tumor endothelial nitric oxide. This in turn can cause vasodilation, increased blood flow and vessel permeability. Alternatively, this early increase in blood flow may reflect a corresponding decrease in the interstitial fluid pressure, which affects the tumor vessel consequentially, the blood flow. ${ }^{21}$ Preferential damage to a sub-population of oxygenated cells may lower the interstitial pressure on micro vessels within the tumor, thus opening capillaries and increasing tumor blood microcirculation. ${ }^{22}$ This effect can facilitate improved chemotherapy delivery to tumors as has been demonstrated in pre-clinical xenograft models. ${ }^{23}$

Figures 3 and 4 show relative intrinsic fluorescence spectra of adenomatous oral tissue and of 4 normal tissue sites. The data was obtained by using the measured fluorescence and reflectance and the values were inserted into Equation 1 in order to extract the intrinsic fluorescence. The four polyps can easily be distinguished from the normal sites by their broadened line shape.

$$
f_{x m}=\frac{F_{x m}}{\frac{1}{\mu_{s x} \ell} \sqrt{\frac{R_{0 x} R_{0 m}}{\delta_{x} \delta_{m}}} \frac{R_{x}}{R_{0 x}}\left(\frac{R_{m}}{R_{0 m}}+\delta_{m}\right)}----(1)
$$

Equation (1) establishes a relationship between the intrinsic fluorescence spectrum, $f_{x m}$, the experimentally measured fluorescence spectrum, $\mathrm{F}_{\mathrm{xm}}$, and the diffuse reflectance spectrum, $R_{m}$. This equation was derived to show that fluorescence can be treated in a similar way as the reflectance. For a fluorescence measurement on a sample with the same amount of fluorophore without absorbers and scatters, the intrinsic fluorescence spectrum, $f_{\mathrm{xm}}$, can be obtained.

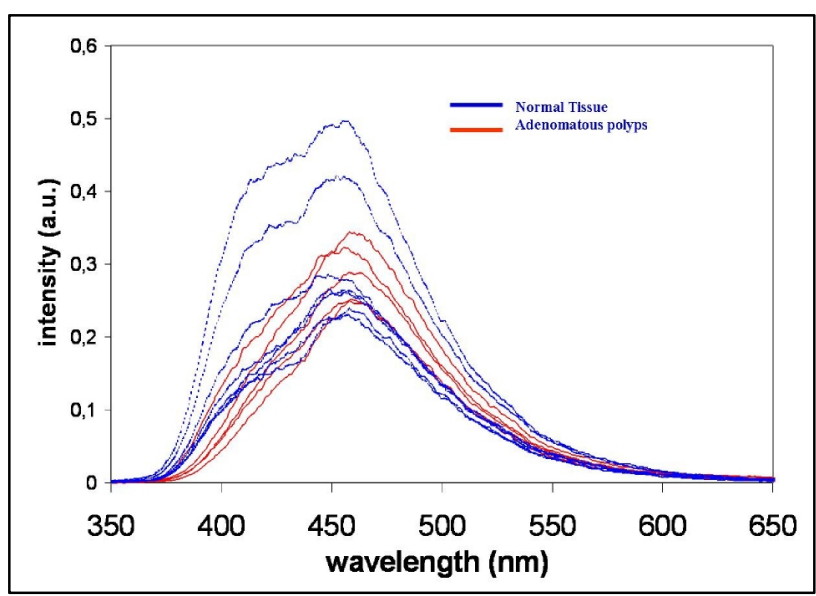

FIG. 3: Fluorescence spectrum of normalized intrinsic fluorescence spectra of 4 adenomatous tissues and 4 normal tissues in oral site

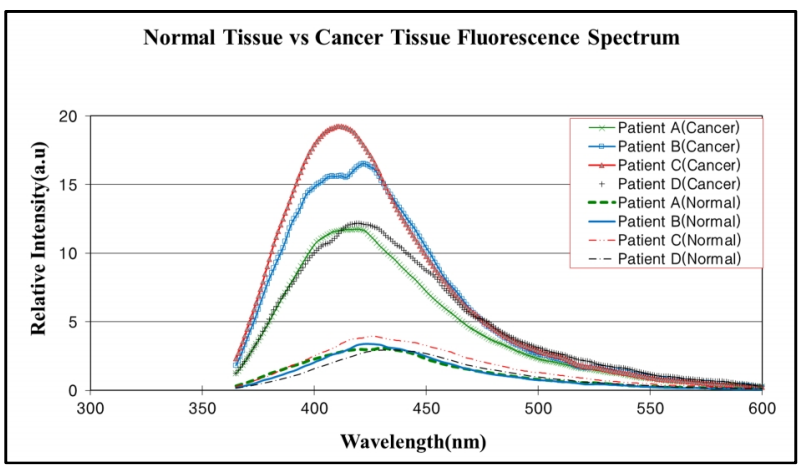

FIG. 4: (a) Fluorescence spectrum of Oral cavity tissue before radiotherapy (4 upper spectrum show the normal tissue, 4 lower spectrum show the cancerous tissue)

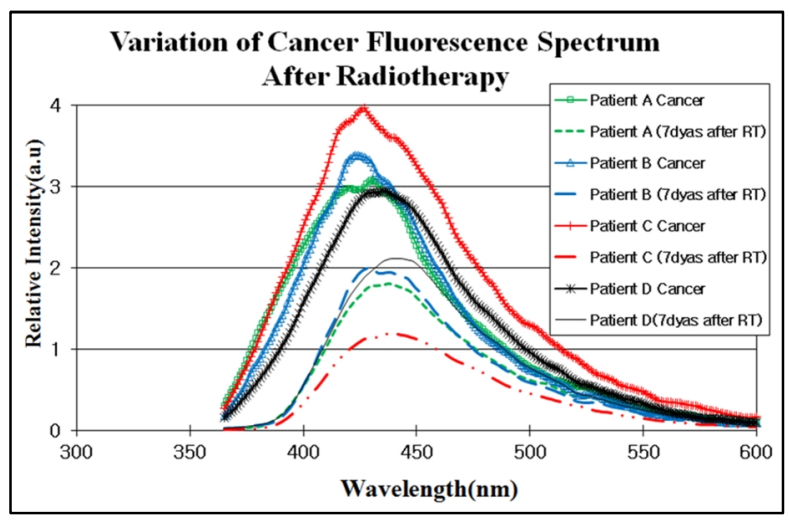
b

FIG. 4: (b) Fluorescence spectrum of Oral cavity tissue after radiotherapy ( 4 weeks) (the 4 lines represent the spectra before radiotherapy and the dashed lines show the results after radiotherapy). 
In Figure 4 (a), Optical fluorescence spectrums showed the possibility of detection of oral cancer as an optical diagnostics tool before radiotherapy treatment. The 4 fluorescence spectra of normal tissue form a line that is above the other 4 spectra that represent the oral cancer tissue. It shows that the fluorescence intensity of cancer tissues is higher than that of the normal tissue. Figure 4 (b) shows the variation of fluorescence spectrum after radiotherapy in 4 patients. All 4 patients who had treatment by radiation for 5 weeks are measured by FastEEM. All fluorescence spectrums intensity after radiotherapy decreased down to the lower after radiotherapy.

\section{Discussion}

In this study, we have demonstrated the feasibility of optical fluorescence spectroscopy as an optical biopsy tool for monitoring oral cancer patients in radiation therapy. First, this was done by using a standard spectrofluorimeter to measure fluorescence EEMs and to determine the location of the endogenous fluorescent peaks and their relative intensity in normal and adenomatous oral tissues for diagnostic in vivo. Next, fluorescence spectra were measured to examine the variations in cancer tissues after radiotherapy. We observed significant differences between the autofluorescence spectra of normal tissues and adenomatous oral tissues.

These differences could be attributed to the differences in fluorophores expression. The endogenous fluorophores that are speculated to be the indicators of cancerous transformations are the amino acids tryptophan and tyrosine, the structural proteins collagen and elastin, the coenzymes NAD (P)H and FAD (Flavin adenine dinucleotide), and phorphyrins. When normal and diseased tissues were compared, the concentration of NADH was higher and the fluorescence intensity was lower in cancerous tissues. This was because the polyp present in abnormal skin made the epidermal layer thicker. However, the second graph of Figure 4 depicts the same portion of cancerous skin before and after treatment. Since the depth of the layer is the same, the fluorescence decreases upon treatment as the NADH level is reduced.

There were some limitations with the current approach and gap for future improvement. In this study, different observers could have possibly applied different probe-tissue pressure and introduced different probe positioning. These effects could have induced variations in quantification, which were quantified and reported as error bars in the figures. In addition, the human oral region consists of different anatomical tissues such as muscle and fat, with its proportion varying across patients. Thus the semi-infinite homogeneous medium approximation is unlikely to be exactly valid. Nevertheless, we have confined to employing the semi-infinite approximation, because it enabled a simplification of analysis and the extraction of trends from the weekly measurements.
Better quantification, as well as increased ability to distinguish different tissue structure (tumor, normal tissue) can be obtained from similar measurements using larger numbers of sources and detectors, and also by image segmentation based on other available anatomical information. ${ }^{24}$

However, in practice, a larger number of sources and detectors also introduces some difficulty interfacing the probe to the tissue. From experience, we have found that the relatively large probe having many fibers was disadvantageous when trying to contact all fibers to the tissue surface with equal pressure. Therefore, a bulkier single probe containing numerous blood flow and oxygen saturation fibers was avoided. In the future a better probe design may enable a better quantification that preserves good probe tissue contact.

Finally, limitations such as uncertainties in tumor boundaries can potentially be eliminated by comparing the optical fluorescence spectroscopy diagnostic methods with other structural imaging modalities such as ultrasound and MRI. Since CT is available only before therapy, adding a structural imaging methodology such as ultrasound to our protocol would enable us to assess tumor size changes weekly during therapy. Tissue heterogeneity effects may also be investigated with imaging techniques.

In this study we have primarily focused on the changes of fluorescence spectrum responses of the tumor that result from radiation therapy. Since the assessment of early response could potentially improve treatment outcome, the results we have presented encourage one to focus on early weeks. Our results suggest that early clinical tumor response to radiation therapy can be detected and quantified by optical fluorescence spectroscopy. The data clearly start to exhibit significant changes within 14 days of therapy. The early flow changes may be significant in affecting drug delivery efficacy and/or tumor oxygenation during radiation therapy and the early tumor oxygenation changes may be related to tumor response. The responses of 4 oral patients were similar to each other, but were different from those of the partial responder. Nevertheless, our statistics are not sufficient to draw significant physiological conclusions. Since a primary aim of therapy diagnostics is to predict the response as early as possible, the early blood flow and oxygenation changes observed here suggest the potential utility of daily measurements during the 14 days of treatment. Since the detection of differences between tumor and normal tissue in vivo, most of optical diagnostics methods have advantage in the field of early cancer detection and for daily based cancer therapy including monitoring of the outcomes of radiotherapy.

\section{Conclusion}

Medical imaging techniques such as MRI, CT and PET are being used as monitoring tools in tumor therapies (radio- 
therapy, chemotherapy, and gene-therapy). However, some limitations include high measurement costs and time consumption. Hence most oncologists may not be able to monitor the effect or variation of tumor and normal tissues surrounding the tumor during therapy.

This study demonstrates the applicability of fluorescence spectroscopy in the monitoring tumor radiotherapy of oral cancer. It was proven that the fluorescence spectroscopic diagnostic technique can detect the difference between premalignant lesion and squamous cell carcinoma of oral cavity by measuring the fluorescence intensity variation in fluorophore NADH in tissue after radiotherapy.

We have quantified tumor changes non-invasively during radiotherapy using the optical fluorescence spectroscopy with FastEEM. These techniques do not require contrast agent administration and are suitable for bedside examinations with rapid data acquisition. Our preliminary data showed that patients exhibit significant changes in $\mathrm{rBF}, \mathrm{SO} 2$, THC and fluorescence spectrum even in the 4 week course of the treatment. In one patient, a different trend was observed with a preliminary indication that it coincided with a different treatment outcome. This odd observation should be further studied with better statistics. At this point, it is difficult to determine what prognostic role these techniques will play in the future. More statistical data are required for assessment of the prognostic value of these optical diagnostics technology. However, it can be expected that the fluorescence spectroscopy as one of optical diagnostics will be a useful tool in monitoring tumor treatment in radiotherapy.

\section{Conflict of interest}

The authors declare that they have no conflicts of interest. The authors alone are responsible for the content and writing of the paper.

\section{References}

1. Mantyla MJ, Toivanen JT, Pitkanen MA, Rekonen AH. Radiation-induced changes in regional blood flow in human tumors. Int J Radiat Oncol Biol Phys 1982; 8:1711-7.

2. Sonveaux P, Brouet A, Havaux X, et al. Irradiation-induced angiogenesis through the up-regulation of the nitric oxide pathway: implications for tumor radiotherapy. Cancer Res 2003; 63:1012-9.

3. Richards-Kortum R, Sevick-Muraca E. Quantitative optical spectroscopy for tissue diagnosis. Annu Rev Phys Chem 1996; 47:555-606.

4. Eker C, Lund Institute of Technology, Lund University, Sweden. Optical characterization of tissue for medical diagnostics. Dissertation thesis, 1999.
5. Georgakoudi I, Jacobson BC, Van Dam J, et al. Fluorescence, reflectance, and light-scattering spectroscopy for evaluating dysplasia in patients with Barrett's esophagus. Gastroenterology 2001; 120:1620 - 9 .

6. Klinteberg C af, Nilsson AMK, Wang I, et al. Laser-induced fluorescence diagnostics of basal cell carcinomas of the skin following topical ALA application. Biomed Opt Newsletter 1996; 5: 1-6.

7. Deckelbaum LI, Lam JK, Cabin HS, et al. Discrimination of normal and atherosclerotic aorta by laser-induced fluorescence. Lasers Surg Med 1987; 7:330-5.

8. Keijzer M, Richards-Kortum RR, Jacques SL, Feld MS. Fluorescence spectroscopy of turbid media: Autofluorescence of the human aorta. Appl Opt 1989; 28:4286-92.

9. Andersson-Engels S, Johansson J, Svanberg K, Svanberg S. Fluorescence imaging and point measurements of tissue: applications to the demarcation of malignant tumors and atherosclerotic lesions from normal tissue. Photochem Photobiol 1991; 53:807-14.

10. Na R, Stender IM, Wulf HC. Can autofluorescence demarcate basal cell carcinoma from normal skin? A comparison with protoporphyrin IX fluorescence. Acta Derm Venereol 2001; 81:246-9.

11. Andersson-Engels S, Johansson J, Stenram U, et al. Diagnosis by means of fluorescent light emission from tissue. Swedish Patent No. 5, 1989.

12. Andersson-Engels S, Johansson J, Svanberg S, Svanberg K. Fluorescence diagnosis and photochemical treatment of diseased tissue using lasers: Part II. Anal Chem 1990; 62:19A-27A.

13. Andersson-Engels S, Johansson J, Stenram U, et al. Malignant tumor and atherosclerotic plaque diagnosis using laser-induced fluorescence. IEEEJ Quant Electr 1990; 26:2207-17.

14. Andersson-Engels S, Baert L, Berg R, et al. Fluorescence characteristics of human atherosclerotic plaque and malignant tumors. Optical methods for tumor treatment and early diagnosis: Mechanisms and Techniques, Proc SPIE 1991; 1426: 31-43.

15. Padhani AR, Ollivier L. The RECIST (Response Evaluation Criteria in Solid Tumors) criteria: implications for diagnostic radiologists. Br J Radiol 2001; 74:983-6.

16. Zhang Q Müller MG, Wu J, Feld MS. Turbidity-free fluorescence spectroscopy of biological tissue. Opt Lett 2000; 25:1451-3.

17. Zonios G, Perelman LT, Backman V, et al. Diffuse reflectance spectroscopy of human adenomatous colon polyps in vivo. Appl Opt 1999; 38:6628-37.

18. Perelman LT, Backman V, Wallace M, et al. Observation of Periodic Fine Structure in Reflectance from Biological Tissue: A New Technique for 
Measuring Nuclear Size Distribution. Phys Rev Lett 1998; 80:627-30.

19. Vaupel P, Kallinowski F, Okunieff P. Blood flow, oxygen and nutrient supply, and metabolic microenvironment of human tumors: a review. Cancer Res 1989; 49:6449-65.

20. Feldmann HJ, Molls M, Vaupel P. Blood flow and oxygenation status of human tumors. Clinical investigations. Strahlenther Onkol 1999; 175:1-9.

21. Milosevic MF, Fyles AW, Hill RP. The relationship between elevated interstitial fluid pressure and blood flow in tumors: a bioengineering analysis. Int J Radiat Oncol Biol Phys 1999; 43:1111-23.
22. Meyn RE, Stephens LC, Voehringer DW, et al. Biochemical modulation of radiation-induced apoptosis in murine lymphoma cells. Radiat Res 1993; 136:327-34.

23. Pietras K, Rubin K, Sjoblom T, et al. Inhibition of PDGF receptor signaling in tumor stroma enhances antitumor effect of chemotherapy. Cancer Res 2002; 62:5476-84.

24. Ntziachristos V, Yodh AG, Schnall M, Chance B. Concurrent MRI and diffuse optical tomography of breast after indocyanine green enhancement. Proc Natl Acad Sci USA 2000; 97:2767-72. 